\title{
openheart Identifying barriers to participation in cardiac prevention and rehabilitation programmes via decision tree analysis: establishing targets for remedial interventions
}

\author{
Orna Reges, ${ }^{1,2,3}$ Noa Vilchinsky, ${ }^{4}$ Morton Leibowitz, ${ }^{2,5}$ Abdulrahem Khaskia, ${ }^{3}$ \\ Morris Mosseri, ${ }^{3}$ Jeremy D Kark ${ }^{1}$
}

To cite: Reges 0 ,

Vilchinsky N, Leibowitz M, et al. Identifying barriers to participation in cardiac prevention and rehabilitation programmes via decision tree analysis: establishing targets for remedial interventions. Open Heart 2014;1:e000097. doi:10.1136/openhrt-2014000097

Received 27 February 2014 Revised 24 May 2014 Accepted 15 July 2014

CrossMark

${ }^{1}$ Hebrew University-Hadassah School of Public Health and Community Medicine,

Jerusalem, Israel

${ }^{2}$ Clalit Research Institute,

Tel-Aviv, Israel

${ }^{3}$ Department of Cardiology,

Meir Medical Center,

Kfar-Saba, Israel

${ }^{4}$ Department of Psychology, Bar Ilan University, Ramat Gan, Israel

${ }^{5}$ Department of Cardiology, N.Y.U. School of Medicine, New York, New York, USA

Correspondence to Orna Reges;

orna.reges@gmail.com

\section{ABSTRACT}

Background: Participation rates of patients with acute coronary syndrome (ACS) in efficacious cardiac prevention and rehabilitation programmes (CPRPs) are low, particularly in ethnic minorities. Few studies have evaluated the full array of potential barriers to participation in a multiethnic cohort with identical insurance coverage.

Objective: To assess the hierarchy of multiple barriers (ie, sociodemographic, systemic, illness related, psychological and cultural) to participation in CPRP of Jewish and Arab patients served by a regional hospital in Israel.

Methods: Patients with ACS ( $N=420)$ were interviewed during hospitalisation about potential barriers and subsequently about participation in CPRP. Decision tree analysis determined, hierarchically, the best predictors of participation in CPRP.

Results: Ethnicity was the salient predictor of participation in CPRP $(61.1 \%(95 \% \mathrm{Cl} 55.6 \%$ to $66.5 \%)$ of Jewish patients versus $17.2 \%(95 \% \mathrm{Cl}$ $11.2 \%$ to $24.9 \%$ ) of Arab patients). Among Jewish patients the dominant determinant was a recommendation for CPRP in the hospital discharge letter $(32.5 \%(95 \% \mathrm{Cl} 23.1 \%$ to $43.1 \%)$ vs $71.9 \%$ (95\% Cl $65.8 \%$ to $77.6 \%$ ) participation without and with a recommendation, respectively). Other major hierarchical determinants included age, discharge diagnosis, socioeconomic position and perceived benefits of exercise. Among Arab patients, anxiety was the main predictor $(5.5 \%(95 \% \mathrm{Cl} 1.1 \%$ to $14.1 \%)$ vs $27.9 \%$ (95\% Cl $17.7 \%$ to $40.0 \%$ ) participation among those with high vs lower anxiety levels). Additional contributors were a predischarge visit to the rehabilitation centre (familiarisation) and car ownership (access).

Conclusions: Utilisation of decision tree analysis enables us to identify the key barriers to participation in CPRP in an ethnic-specific mode. Interventions to improve participation can then be designed to address each group's specific barriers.

\section{KEY MESSAGES}

What is already known about this subject?

- Multiple causes have been identified to account for low participation rates in cardiac rehabilitation.

What does this study add?

- Establishes an hierarchal assessment of barriers to participation utilizing decision tree analysis to identify critical decision nodes.

How might this impact on clinical practice?

- Attempts to augment patient participation rates require an understanding of critical decision nodes so that resources for improvement can be appropriately allocated.

\section{INTRODUCTION}

In order to prevent the recurrence of acute coronary syndrome (ACS), ${ }^{1-3}$ cardiac prevention and rehabilitation programmes (CPRPs) are now an inherent element in the European, ${ }^{45}$ American $^{67}$ and Israeli ${ }^{8}$ formal guidelines for secondary prevention following ACS.

Unfortunately, despite the proven efficacy of CPRP, ${ }^{9-11}$ there is a substantial underutilisation of this service worldwide. ${ }^{12-15}$ Therefore, the identification of putative barriers to CPRP participation has been the focus of our research team in Israel in the past several years. ${ }^{16-19}$

In our former publications, we identified sociodemographic, systemic and cognitive barriers to participation in CPRP. Overall, ethnicity was found to be the salient barrier with significantly lower participation among Arab patients compared with Jewish patients. Despite accumulated evidence pointing at ethnicity as a key factor in explaining underutilisation of health 
services, cultural values putatively underlying the association between ethnicity and CPRP adherence have not been previously investigated. Therefore, in the current study we measured specific illness-related cultural world views. Also included were the psychological manifestations of anxiety and depression that others have detected as barriers for CPRP participation. ${ }^{20} 21$

We have also identified non-cultural barriers including lower socioeconomic position (SEP), history of coronary heart disease (CHD), diagnosis of unstable angina pectoris (UAP) (compared with myocardial infarction (MI)), absence of a recommendation to participate in CPRP in the discharge letter, absence of a visit to the rehabilitation centre during hospitalisation, the lack of a driving license, ${ }^{16}{ }^{17}$ high levels of anxiety and depression, ${ }^{18}$ low perceived benefits of regular exercise, and a low sense of personal control. ${ }^{19}$ Although we were able to identify those predictors of participation in CPRP, our previous reports did not evaluate the relative contribution of each barrier, and also did not provide clinically relevant cut-off points at which each variable becomes a barrier, a prerequisite for any serious endeavour of tailoring interventions for the most in-need populations. Therefore, in the current study we analyse, in a comprehensive manner, all the putative barriers to participation in CPRP evaluated in the cohort, including the cultural and psychological aspects.

Our objectives were (1) to determine the hierarchy of the strengths of each of the above-mentioned barriers to CPRP participation and (2) to determine dichotomous cut-off points at which any of the predictor variables becomes a significant barrier to CPRP participation.

\section{METHODS}

\section{Study population}

The methods of the current study have been previously reported. ${ }^{16-19} 22$ The study was undertaken at a regional hospital in central Israel serving both Jewish and Arab populations. The hospital provides an in-house comprehensive cardiac prevention and rehabilitation programme with a strong emphasis on physical activity. In brief, 1705 patients admitted to the coronary care unit (CCU) of the Meir medical centre or transferred from the internal medicine wards to the CCU for urgent catheterisation, between January 2009 and August 2010, were registered. Of these, 19 died during hospitalisation and 603 were discharged with no ACS diagnosis. Of the remaining 1083 patients, 434 were not eligible for the study due to the following criteria: having severe physical/mental disability that would prevent participation in CPRP (244), early referral for further acute phase treatment at another hospital (98), inability to be interviewed in Hebrew or Arabic (52) and inability to commit to the study $(n=40)$. Of the 649 eligible patients, 501 consented to be interviewed during hospitalisation (77.2\% response rate); of these $83.8 \%$ consented to be interviewed at the 6-month follow-up (for a flow diagram, see figure 1 in our previous publication: ref. 21). The final study sample comprised 420 patients who had been hospitalised due to ACS (as confirmed by a senior cardiologist based on positive electrocardiographic changes or positive troponin levels) and who were eligible to participate in CPRP. All the respondents were interviewed in their native tongue (Hebrew or Arabic). No differences were found in response rate according to age or ethnic group. There were, however, differences according to gender and diagnosis with higher response rates among men than women $(67.5 \%$ and $52.8 \%$, respectively) and between patients with MI versus patients with UAP $(68.6 \%$ and $56.7 \%$, respectively). The mean age of the respondents was 59.6 \pm 10.9 years, $84.5 \%$ were men and $15.5 \%$ were women, $72.4 \%$ were Jews and $27.6 \%$ were Arabs, and $71.7 \%$ were hospitalised for AMI and 28.3\% for UAP. Informed consent was obtained from each patient. The study was authorised by the hospital Institutional Review Board.

\section{Variable definitions}

Data regarding the main outcome, participation in CPRP (coded as yes/no), were collected in the follow-up interview. Patients who indicated that they had joined any long-term CPRP after the index event, at the Meir medical centre or elsewhere for a period of at least 1 month were classified as participants. Among the potential barriers specifically examined were as below:

Sociodemographic characteristics, which included ethnicity (Jews/Arabs), gender, age, birth place (Israel/other), marital status, level of education (highest certificate/ degree earned), employment status (gainfully employed or not), economic situation (subjective assessment), religiosity, health services supplier membership and subjective SEP. $^{1922}$

Baseline clinical data that were extracted from patient medical records included the hospitalisation unit (CCU/Internal medicine), diagnosis (MI/UAP), personal history of CHD (yes/no) and traditional CHD risk factors (family history of CHD, obesity, smoking, dyslipidemia, diabetes and hypertension (yes/no-unknown, for all risk factors)).

System-based barriers included access-based barriers namely no driving license, not in possession of a car and limited familiarity with Hebrew, and hospital-based barriers namely absence of a recommendation to participate in CPRP in the discharge letter and absence of a documented visit to the rehabilitation centre during hospitalisation, a routine part of the intensive recruitment programme. ${ }^{17}$

We emphasise that the only eligibility criteria for inclusion in the study, in addition to informed consent, were physical and emotional suitability for joining CPRP. There were no other declared criteria for preventing patients from visiting the CPRP or being referred to the programme.

Illness cognition barriers included a health beliefs component and a control component. ${ }^{19}$ The health beliefs component was measured using the 28-item 
questionnaire developed by Mirotznik et $a l^{23}$ The control component was measured by the cure/control dimension of the revised Illness Perception Questionnaire. $^{24}$

Psychological barriers included anxiety (6 items) and depression (7 items) ${ }^{25}$ of the validated Brief Symptom Inventory. ${ }^{26}$ Responses for level of distress over the past days ranged from 1 (not at all) to 5 (extremely). The average score for each subscale was used.

Cultural barriers were conceptualised as specific illnessrelated cultural world views. These were measured using a 34-item scale designed specifically for the purpose of the current study, based on Cohen and Azaiza's ${ }^{27}$ Arab Culture-Specific Barriers questionnaire, and on in-depth interviews tapping the issue of coping with cardiac illness, which were held with focus-groups of patients and service providers of the two ethnic groups.

\section{Data analysis}

Decision tree analysis, a data mining technique, was used to create set of rules for predicting participation in CPRP. $^{28}$ The decision tree is represented by a flow chart consisting of nodes that split and create branches. At each stage (node) the best predictor for participation in CPRP was selected and the optimal forecasting values were determined. The Chi Squared AID (CHAID) algorithm ${ }^{29} 30$ was used; the number of splits was limited to 4 and the number of patients in a specific node was restricted to 20 and above to limit the number of branching points and preserve statistical power.

Data for the various variables included in the decision tree analysis were complete for $87.1 \%$ of the patients. As the extent of missing data was less than $5 \%$ for each variable separately, multiple imputations were not used. Data for the variables actually included in the decision tree (presented in figure 1) were complete for $93.6 \%$ of the patients. As per the CHAID algorithm used, missing values are included in the tree-growing process as a floating category that is allowed to merge with other categories at tree nodes.

To validate the present decision tree, a fivefold crossvalidation procedure for decision tree analysis available on SPSS statistical software was used. In short, a series of decision trees is generated, each time excluding onefold of the sample (one-fifth). Misclassification risk is then calculated by applying the tree to the excluded fold and identifying the number of cases that are incorrectly classified. The following estimates were calculated (1) a misclassification risk estimate, calculated on the complete sample; (2) an overall accuracy percentage and (3) the cross-validation risk estimate.

The risk estimate for the classification tree was 0.23. The classification tree analysis accurately predicted patients' participation in CPRP $(77 \%$ correct $)$. The cross-validation estimate for risk of misclassification (0.27) approximated that of the full model (0.23) .

\section{RESULTS}

Table 1 in our previous publication ${ }^{22}$ presents the description of the main patient characteristics according to population group.

Utilising decision tree analysis (figure 1), the single most critical element determining participation in CPRP was ethnic background. The majority of the Jewish patients, that is, $61.1 \%$ (95\% CI $55.6 \%$ to $66.5 \%$ ), participated in a CPRP compared with less than a fifth of the Arab patients, that is, $17.2 \%$ (95\% CI $11.2 \%$ to $24.9 \%$ ).

Within the Jewish patients the most important contribution to participation was the presence of a written recommendation in the discharge letter from the hospital to their personal physicians. Only $32.5 \%$ (95\% CI $23.1 \%$ to $43.1 \%$ ) of patients not having such a recommendation participated as opposed to the $71.9 \% \quad(95 \%$ CI $65.8 \%$ to $77.6 \%$ ) participation rate when such a recommendation was present. The other major contributors were age, discharge diagnosis, SEP, the health services supplier and perceived benefits of exercise. When no recommendation was included in the discharge letter and patients were above age 74 years only $8.3 \%$ (95\% CI $1.4 \%$ to $24.9 \%$ ) participated in CPRP compared with a $42.4 \%(95 \%$ CI $30.3 \%$ to $55.2 \%$ ) participation rate among those aged 74 years or younger who also lacked a documented recommendation. This decision node was, however, affected by the discharge diagnosis with $58.3 \%$ (95\% CI $36.7 \%$ to $77.9 \%$ ) of patients with an MI participating as opposed to $31.4 \%$ (95\% CI $17.8 \%$ to 48.1 ) with a discharge diagnosis of UAP. Among Jewish patients who did receive a recommendation for participation in the discharge letter, SEP came up as a crucial determinant for participation with an $80.6 \%$ (95\% CI $73.5 \%$ to $86.4 \%$ ) participation rate among high SEP patients. Among Jewish patients of lower SEP, health beliefs proved important with a $90.5 \%$ (95\% CI $72.0 \%$ to $98.4 \%$ ) participation rate in the subgroup who professed a belief that physical exercise was a critical element in preventing acute cardiac events.

In the Arab patients anxiety played an important role; there was $27.9 \%$ (95\% CI $17.7 \%$ to $40.0 \%$ ) participation among those with moderate or low anxiety levels as contrasted with $5.5 \%$ (95\% CI $1.1 \%$ to $14.1 \%$ ) among those with high anxiety levels. Further branching was precluded by the small number of Arab participants. To evaluate possible additional determinants anxiety was removed and the decision tree analysis was recomputed (figure 2). Excluding anxiety, the importance of the systemic barriers of a rehabilitation centre visit prior to discharge and possession of a car became evident.

\section{DISCUSSION}

The current study was undertaken to establish a hierarchy of barriers to patients' participation in CPRP. Following Balady's mapping, ${ }^{31}$ we incorporated into the model variables related to sociodemographic, systemic, clinical and cognitive dimensions, many of which have 


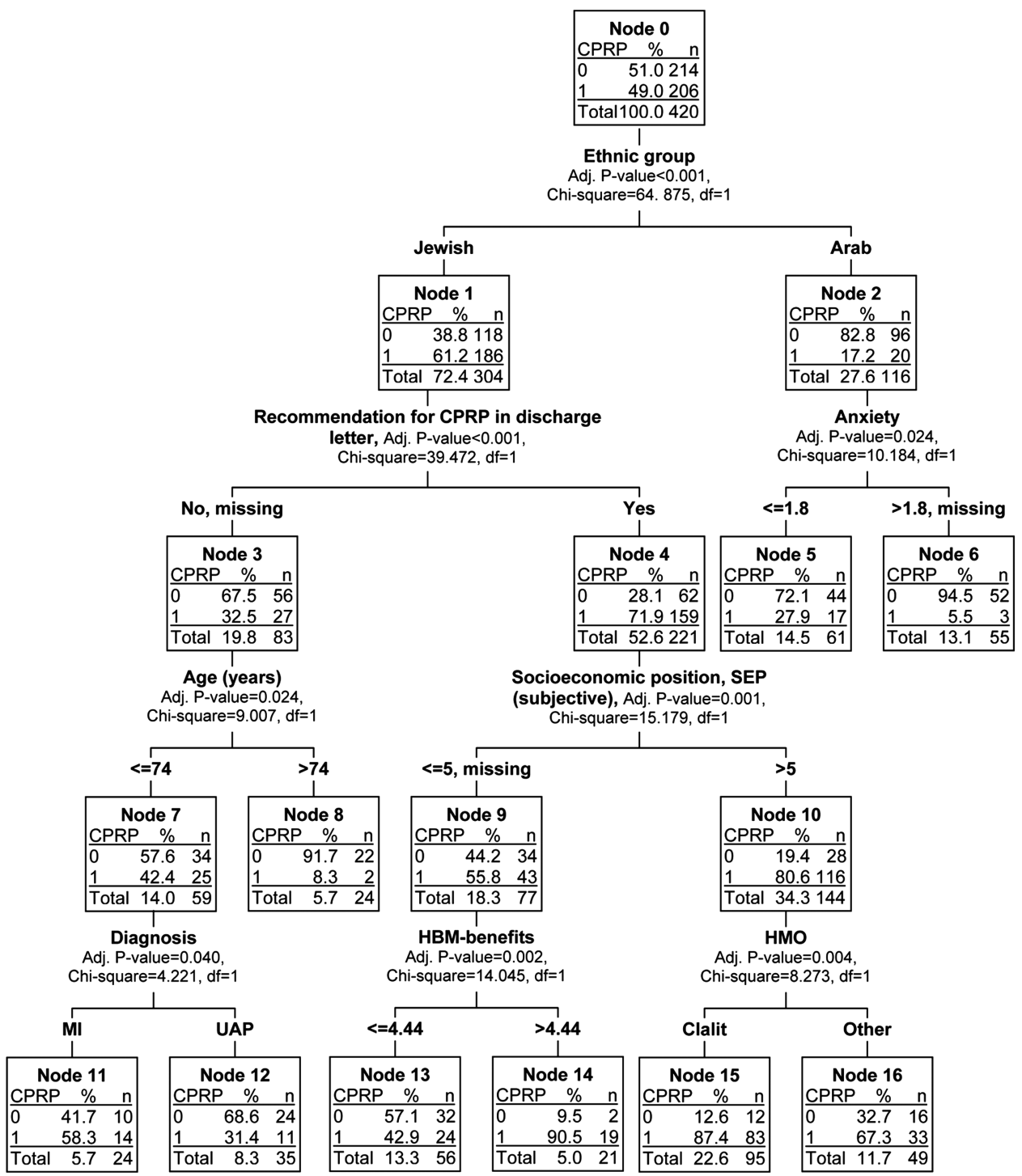

Figure 1 Decision tree (using the CHAID algorithm) for predicting participation in a cardiac prevention and rehabilitation programme (CPRP) among 420 Jewish and Arab patients with acute coronary syndrome (ACS). At each node the best predictor for participation in CPRP was selected from multidimensional potential barriers and the optimal forecasting values were determined (CPRP categories: $0=$ did not participate in CPRP, $1=$ participated in CPRP).

been demonstrated in smaller less comprehensive studies to play a role, as well as psychological manifestations and illness-related cultural world views. A decision tree analyses were implemented to identify not only which variables are significant barriers but also to establish hierarchal rankings of their importance in these two ethnic groups.

Overall, ethnicity (Arab minority) was by far the most important barrier to participation. When analysing the barriers for each ethnic group separately, a distinct picture emerged for each group. For Jewish patients, receiving a recommendation to participate in CPRP was associated with an increased probability of participation. Among those who did receive such a recommendation, low SEP became a barrier to participation. Narrowing down to those with low SEP, low levels of belief in the benefits of such intervention were identified as the main obstacle.

Among Jewish patients who did not receive a recommendation to participate in CPRP, age was found to be an important determinant. With age introduced as a continuous variable, the decision tree analysis identified age 74 years as the point at which it becomes a significant barrier. This vulnerable subgroup, characterised by a very low probability to participate $(8.3 \%, 95 \%$ CI $1.4 \%$ to $24.9 \%$ ) represents the older patients who were discharged from hospital with no written recommendation. Given the documented benefit of CPRP in older patients, ${ }^{32}$ the recommendation becomes particularly important for this subgroup.

Among Arab patients, the overwhelmingly important determinant was level of anxiety with a probability of 


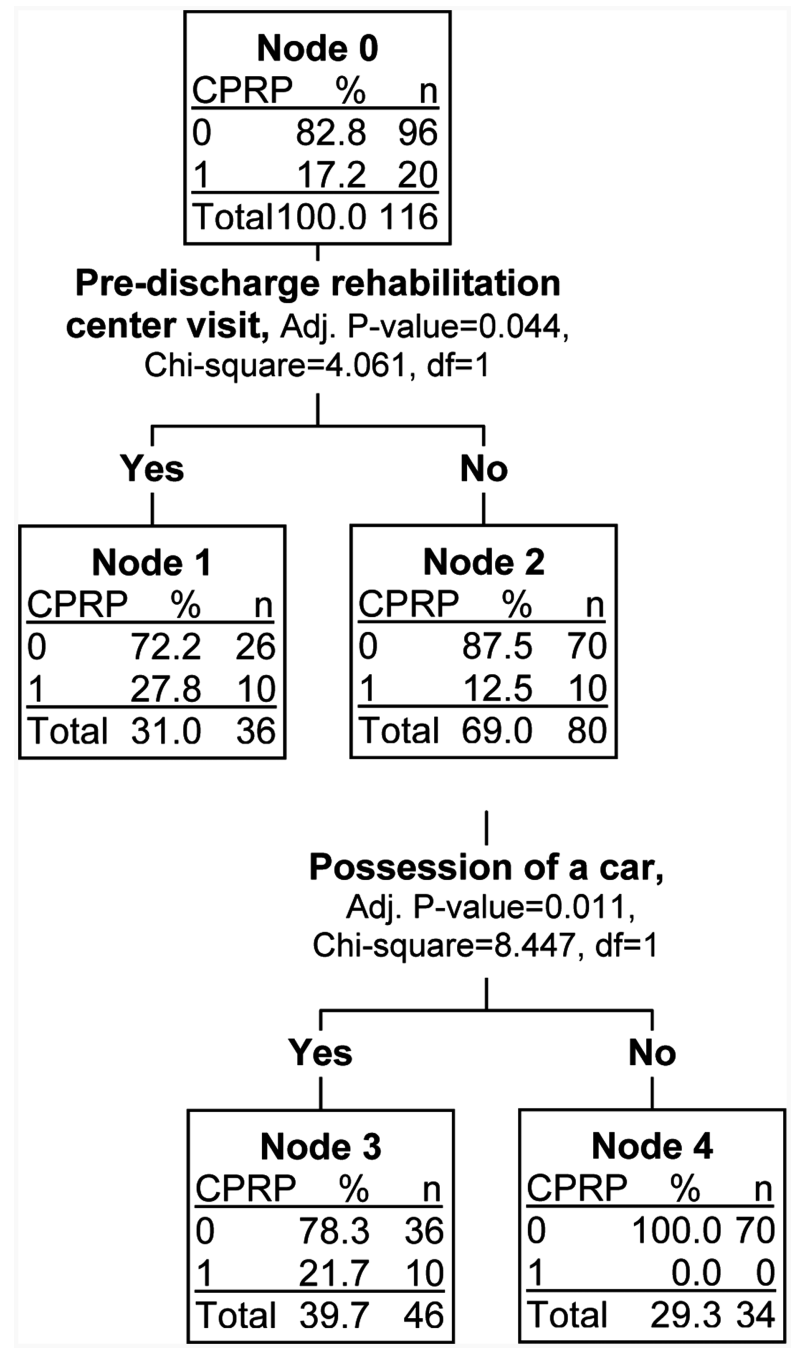

Figure 2 Decision tree (using the CHAID algorithm) for predicting participation in a cardiac prevention and rehabilitation programme (CPRP) restricted to the 116 Arab patients with ACS patients. At each node the best predictor for participation in CPRP was selected from multidimensional potential barriers and the optimal forecasting values were determined (CPRP categories: $0=$ did not participate in CPRP, $1=$ participated in CPRP).

just $5.5 \%$ (95\% CI $1.1 \%$ to $14.1 \%$ ) to attend when the level of anxiety was higher than 1.8 (based on the Brief Symptoms Inventory questionnaire) vs $27.9 \%$ (95\% CI $17.7 \%$ to $40.0 \%$ ) when the level was lower than 1.8 . These results provide an important focal point for further studies. Focusing on these specific barriers, and analysing the main barriers within each ethnic group, may prove more beneficial in designing interventions.

Our analysis represents an important addition to the understanding of barriers to participation in CPRP. The roles of ethnicity, letters of recommendation, age and SEP are already well established in the literature. The confirmation of their importance and their incorporation and positioning into the decision tree hierarchy in the analysis of the Jewish sector is an observation that will facilitate the implementation of remedial interventions. For example, SEP, although an established barrier, is a variable that is difficult if not impossible to amend; therefore, efforts should be channelled into improving the recommendation letter procedure as well as to the augmenting of belief in the benefits of CPRP. Especially among the Jewish sector, focusing on these barriers may prove more beneficial than anything else.

The analysis in the Arab sector is more limited because of the constraints of the sample size, including a very small number of female participants. This is the main limitation of our study. Nevertheless, the ranking of anxiety levels as the most important barrier in the Arab sector provides an important focal point for further investigation. In addition, efforts should be channelled into minimising those systemic barriers that are mostly relevant for the Arab patients. Making a predischarge visit to the rehabilitation centre an integral and routine part of a patient's hospitalisation for ACS as well as providing each eligible patient a mandatory recommendation letter for joining their local CPRP may be useful in facilitating participation, especially among this minority group. Our former findings based on the same database, which showed that absence of a letter of recommendation is a strong barrier for CPRP attendance beyond all other sociodemographic and clinical variables, ${ }^{17}$ strengthen this inference. It is important to further decipher the determinants of these systemic barriers between the two ethnic groups. The fact that car ownership came up as a predictor among the Arab patients indicates that access is a topic that should be addressed. Although unique to our study is an attempt to identify the specific illness-related cultural world views which might account for the overwhelming differences in participation rates between the two ethnic groups studied, none of the components assessed came up as significant barriers when integrated into the decision tree analysis.

In summary, in 2011, the AHA issued a presidential advisory related to referrals to and participation in programmes of CPRP. ${ }^{31}$ They include a list of 25 factors which have been identified as contributing to nonparticipation. Our study incorporated many of those factors into the evaluation of barriers in one regional hospital serving an ethnically and economically diverse population and confirmed many of the AHA recommendations. By establishing a hierarchy of importance, however, we enable more targeted interventions directed to specific subpopulations to improve attendance. The recognition that age, discharge diagnosis, health beliefs and, arguably most importantly, anxiety and letters of referral are critical elements in promoting participation is reinforced by our analysis. Our hierarchy of barriers, however, emphasises the over-riding importance of ethnicity and minority status and places strong focus on the importance of evaluating barriers specific to different cultural groups. ${ }^{33-35}$ 
Contributors OR participated in the creation of the study's concept and design, and organised and took part in the data collection, carried out statistical analysis, took part in the interpretation of the data, and drafted the manuscript. NV participated in the creation of the study's concept and design, provided advice and guidance on statistical and psychological issues, took part in the interpretation of the data, and critically revised the draft. ML participated in the creation of the study's concept and design, supervised the data collection from a medical perspective, took part in the interpretation of the data, critically revised the draft, and helped obtain funding. AK contributed to the conception of the study, provided medical advice during the data acquisition/collection phase, and helped in the design of the questionnaires. MM took part in the interpretation of the data, critically revised the draft, supervised the study and obtained funding. JDK contributed to the study's conception and design, advised on statistical and epidemiological issues, took part in the interpretation of the data, and critically revised the draft. All authors read and approved the final manuscript.

Funding This study was supported by grants from the Israel National Institute for Health Policy and Health Services Research (NIHP) (grant number - 2005/ $113 / \chi)$ and the Israel Heart Fund.

Competing interests None.

Ethics approval Meir Medical Center IRB.

Provenance and peer review Not commissioned; externally peer reviewed.

Data sharing statement No additional data are available.

Open Access This is an Open Access article distributed in accordance with the Creative Commons Attribution Non Commercial (CC BY-NC 3.0) license, which permits others to distribute, remix, adapt, build upon this work noncommercially, and license their derivative works on different terms, provided the original work is properly cited and the use is non-commercial. See: http:// creativecommons.org/licenses/by-nc/3.0/

\section{REFERENCES}

1. Lozano R, Naghavi M, Foreman K, et al. Global and regional mortality from 235 causes of death for 20 age groups in 1990 and 2010: a systematic analysis for the Global Burden of Disease Study 2010. Lancet 2012;380:2095-128.

2. Goldberger N, Aburbeh M, Haklali Z. Leading causes of death in Israel 2000-2010. Ministry of health, Heallth Informatiion Department (Hebrew). 2013.

3. Yeh RW, Sidney S, Chandra M, et al. Population trends in the incidence and outcomes of acute myocardial infarction. $N$ Engl J Med 2010;362:2155-65.

4. Van de Werf F, Bax J, Betriu A, et al. Management of acute myocardial infarction in patients presenting with persistent ST-segment elevation: the Task Force on the Management of ST-Segment Elevation Acute Myocardial Infarction of the European Society of Cardiology. Eur Heart J 2008;29:2909-45.

5. Piepoli MF, Corra U, Adamopoulos S, et al. Secondary prevention in the clinical management of patients with cardiovascular diseases. Core components, standards and outcome measures for referral and delivery. Eur J Prev Cardiol 2012;21:664-81.

6. Antman EM, Anbe DT, Armstrong PW, et al. ACC/AHA guidelines for the management of patients with ST-elevation myocardial infarction: a report of the American College of Cardiology/American Heart Association Task Force on Practice Guidelines (Committee to Revise the 1999 Guidelines for the Management of Patients with Acute Myocardial Infarction). Circulation 2004;110:e82-292.

7. Wenger NK. Current status of cardiac rehabilitation. J Am Coll Cardiol 2008;51:1619-31.

8. Israel Health Ministry. Order No. 54/2006 Jerusalem December 26th 2006.

9. Lawler PR, Filion KB, Eisenberg MJ. Efficacy of exercise-based cardiac rehabilitation post-myocardial infarction: a systematic review and meta-analysis of randomized controlled trials. Am Heart $J$ 2011;162:571-84.e2

10. Taylor RS, Brown A, Ebrahim S, et al. Exercise-based rehabilitation for patients with coronary heart disease: systematic review and meta-analysis of randomized controlled trials. Am J Med 2004;116:682-92.
11. Pack QR, Goel K, Lahr BD, et al. Participation in cardiac rehabilitation and survival following coronary artery bypass graft surgery: a community based study. Circulation 2013; 128:590-7.

12. Suaya JA, Shepard DS, Normand SL, et al. Use of cardiac rehabilitation by Medicare beneficiaries after myocardial infarction or coronary bypass surgery. Circulation 2007;116:1653-62.

13. Mochari $\mathrm{H}$, Lee JR, Kligfield $P$, et al. Ethnic differences in barriers and referral to cardiac rehabilitation among women hospitalized with coronary heart disease. Prev Cardiol 2006;9:8-13.

14. Ades PA, Waldmann ML, Polk DM, et al. Referral patterns and exercise response in the rehabilitation of female coronary patients aged greater than or equal to 62 years. Am J Cardiol 1992;69:1422-5.

15. Allen JK, Scott LB, Stewart KJ, et al. Disparities in women's referral to and enrollment in outpatient cardiac rehabilitation. J Gen Intern Med 2004:19:747-53.

16. Reges O, Vilchinsky N, Leibowitz M, et al. Arab-Jewish differences in attending cardiac rehabilitation programs following acute coronary syndrome. Int J Cardiol 2013;163:218-19.

17. Reges O, Vilchinsky N, Leibowitz M, et al. Systemic determinants as barriers to participation in cardiac prevention and rehabilitation services after acute coronary syndrome. Int $J$ Cardiol 2013;168:4865-7

18. Reges $\mathrm{O}$, Vilchinsky $\mathrm{N}$, Leibowitz $\mathrm{M}$, et al. Change in health behaviours following acute coronary syndrome: Arab-Jewish differences. In press.

19. Reges $\mathrm{O}$, Vilchinsky N, Leibowitz $\mathrm{M}$, et al. Illness cognition as a predictor of exercise habits and participation in cardiac prevention and rehabilitation programs after acute coronary syndrome. BMC Public Health 2013;13:956.

20. Parkosewich JA. Cardiac rehabilitation barriers and opportunities among women with cardiovascular disease. Cardiol Rev 2008;16:36-52.

21. Taylor GH, Wilson SL, Sharp J. Medical, psychological, and sociodemographic factors associated with adherence to cardiac rehabilitation programs: a systematic review. J Cardiovasc Nurs 2011;26:202-9.

22. Reges $\mathrm{O}$, Vilchinsky $\mathrm{N}$, Leibowitz $\mathrm{M}$, et al. Change in health behaviours following acute coronary syndrome: Arab-Jewish differences. Eur J Prev Cardiol 27 Jan 2014. Epub ahead of print.

23. Mirotznik J, Feldman L, Stein R. The health belief model and adherence with a community center-based, supervised coronary heart disease exercise program. J Community Health 1995;20:233-47.

24. Moss-Morris R, Weinman J, Petrie KJ, et al. The revised illness perception questionnaire (IPQ-R). Psychol Health 2002;17:1-16

25. Gilbar O, Ben-Zur H. Adult Israeli community norms for the Brief Symptom Inventory (BSI). Int J Stress Manag 2002;9:1-10.

26. Derogatis LR, Melisaratos N. The Brief Symptom Inventory: an introductory report. Psychol Med 1983;13:595-605.

27. Cohen M, Azaiza F. Developing and testing an instrument for identifying culture-specific barriers to breast cancer screening in Israeli Arab women. Acta Oncol 2008;47:1570-7.

28. Podgorelec V, Kokol P, Stiglic B, et al. Decision trees: an overview and their use in medicine. J Med Syst 2002;26:445-63.

29. Kass G. An exploratory technique for investigating large quantities of categorical data. Appl Stat 1980;29:119-27.

30. Magidson J. The use of the new ordinal algorithm in CHAID to targetprofitable segments. J Database Mark 1993;1:29-48.

31. Balady GJ, Ades PA, Bittner VA, et al. Referral, enrollment, and delivery of cardiac rehabilitation/secondary prevention programs at clinical centers and beyond: a presidential advisory from the American Heart Association. Circulation 2011;124:2951-60.

32. Pasquali SK, Alexander KP, Peterson ED. Cardiac rehabilitation in the elderly. Am Heart J 2001;142:748-55.

33. Stuart-Shor EM, Berra KA, Kamau MW, et al. Behavioral strategies for cardiovascular risk reduction in diverse and underserved racial/ ethnic groups. Circulation 2012;125:171-84.

34. Valencia HE, Savage PD, Ades PA. Cardiac rehabilitation participation in underserved populations. Minorities, low socioeconomic, and rural residents. J Cardiopulm Rehabil Prev 2011;31:203-10.

35. Jolly K, Greenfield SM, Hare R. Attendance of ethnic minority patients in cardiac rehabilitation. J Cardiopulm Rehabil 2004;24:308-12. 